\title{
Dentistry on the bridge to nanoscience and nanotechnology
}

\section{Marco Salerno * and Alberto Diaspro}

Department of Nanophysics, Istituto Italiano di Tecnologia, Genova, Italy

\author{
Edited by: \\ Partha Pratim Mondal, Indian Institute \\ of Science, India \\ Reviewed by: \\ Jose L. Toca-Herrera, University of \\ Natural Resources and Life Sciences, \\ Austria \\ Shivani Sharma, University of \\ California Los Angeles, USA \\ ${ }^{*}$ Correspondence: \\ Marco Salerno, Department of \\ Nanophysics, Istituto Italiano di \\ Tecnologia, via Morego 30, Genova \\ 1-16163, Italy \\ e-mail: marco.salerno@iit.it
}

\begin{abstract}
Dentistry is the area of medical sciences that is most resistant to the introduction of the novel methods arisen from the development of nanoscience and nanotechnology in the last 20 years. Without moving on to science-fiction-like views pointing to times far ahead in the future, we show that the available nanoscale devices and processes of current science and technology, partly inherited from the areas of microscopy and microelectronics, have already proven to be useful for research and development in different fields of dental research. To this goal, we review some results obtained in the last few years at our Institute in the area of dental materials and their characterization, which showed successful application of our background in microscopy and nanoengineering.
\end{abstract}

Keywords: dental materials, microscopy, surface roughness, nanofabrication, resin composites, implants

\section{THE BROAD SCOPE OF DENTISTRY}

Dentistry is considered to be a branch of medicine, such as orthopedics or physiology or neurology. In fact, while based on common foundations of medicine, dentistry is a large stand-alone area, which has its own grasps to the different fields mentioned above (American Dental Association, 2014). For example, topics of periodontal ligament health and dental implant osteointegration clearly relate to a peculiar "dental orthopedics"; the fact that teeth are not a mineralized appendix but rather a living organ in a living environment (gingiva, oral liquids) involves a specific "dental physiology"; the sensitivity of the living tooth, in connection with the pulp and related nerves, can be associated to a possible "dental neurology." Indeed, the oral environment, which is the place where dental functions are operated, is one of the most complex ones in the human body.

As a result, within the dental area several specialized disciplines exist (see Figure 1). Conservative dentistry aims to maintain the original denture and largely overlaps with restorative dentistry, the latter encompassing prosthodontics, periodontics, and endodontics. Prosthodontics (or prosthetic dentistry) is required in case of massive irreversible tooth damage that cannot be fixed with simple resin composite filling, and requires the use of prosthesis for teeth, namely crowns, bridges, and even whole dentures. Periodontics (or periodontology) addresses the diseases of the periodontum, i.e., the teeth ligaments. Endodontics focuses on therapy of the root canals and connected pulp diseases. Oral surgery relates to the extraction of teeth that cannot be saved anymore and should be replaced by implants, and is usually combined with maxillo-facial surgery, extending the treated area around the denture. Prosthodontics, periodontics, and surgery correlate to implantology, which deals with the installation of dental implants. Orthodontics concerns the alignment and straightening of teeth, as well as fixing midface and mandibular growth issues. Pedodontics (or pediatric dentistry) relates to the specific dental issues of children's temporary dentures. Of course, oral and maxillofacial surgery is accompanied by related pathology and radiology specialties, as well as dental anesthesiology. Similarly, oral biology exists as a field on its own, also extending to craniofacial biology. In addition to the obvious extension to animals in veterinary dentistry, more niche specialties exist such as forensic odontology, using dental evidence in law to document people identity, and geriodontics (or geriatric dentistry), associating the delivery of dental care in old adults with aging issues. In US, Dental public health is yet another specialty, involved with social and political issues of dental therapy and follow-up. Sometimes the term odontostomatology is also found, from Greek "odontos" for teeth and "stomatos" for mouth, which is not a dental specialty but a general term including dental and other non-teeth related diseases such as oral cancer. In Italy, the term gnatology (or stomatognatology) is also common, as the discipline focused on occlusion of maxillary bones and their geometricalfunctional relations to teeth, muscles, and nerves. Dental hygiene has in the recent years become an independent specialty as well.

Such a broad range of topics clearly makes use of a large spectrum of materials. In parallel, advanced technological solutions are required for the application of the underlying scientific principles. Actually, in dentistry, many special processes and practice protocols have been developed over the past century. As a result, dentistry is separated from the other medical areas, and the recent integration of nanotechnology and nanoscience into dentistry is progressing more slowly than for general medicine. The goal of this review is to report about the current application of nanotechnology and nanoscience in dentistry. This work does not pretend to be comprehensive, and will address sparse examples mainly from the fields of restorative materials and implantology, where we envisage the highest chances of successful application of nanoscience and nanotechnology. Additionally, the chance of having pharmaceutical treatment and diagnostics will be mentioned shortly. After reading this work, it should be clear that a more 


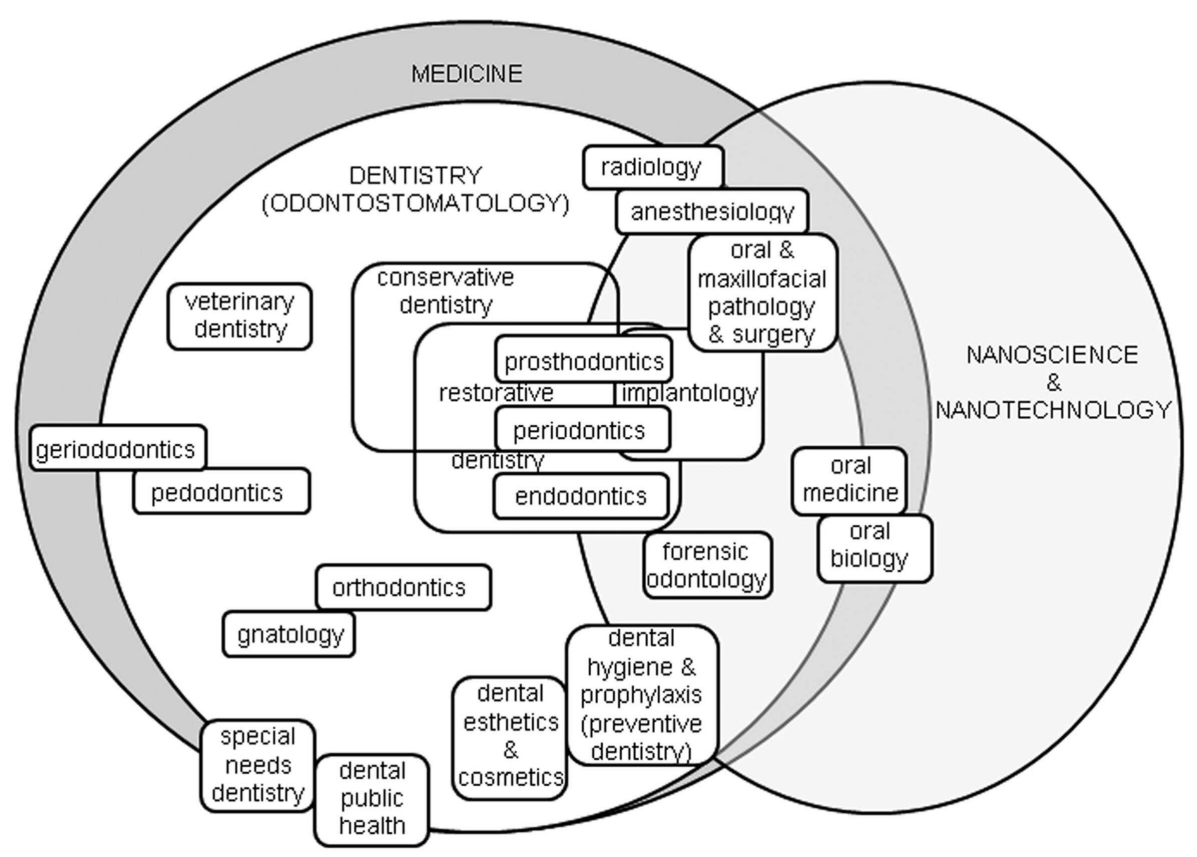

FIGURE 1 |The dental arena, roughly contained in the area of medicine, and its specialties most likely connected to the advances in nanoscience and nanotechnology.

thorough application of concepts and tools emerged in the last two decades in nanoscience and nanotechnology could make dentistry benefit significantly and progress faster in the close future.

\section{NANOTECHNOLOGY AND NANOSCIENCE IN MEDICINE: NOT JUST SCIENCE FICTION}

The Drexler's view (Freitas, 2000; Kumar and Vijayalakshmi, 2006), with billions of colloidal nanorobots injected in sick organ tissue or its environment (the mouth here) and working independently, is not under discussion in this work. The limitations of this utopia (such as the problem of producing and programing these nanounits efficiently, and having them move in a realm where adhesion forces overcome macroscale ones such as gravity) are well-known and not yet successfully addressed. Since we want to avoid approaches still too far from reality, we are not considering what could eventually be done in 20 years. This review describes common topics in dentistry addressed recently by nanotechnological tools with at least partial success. In particular, we will focus on the probably most important nanotechnological tool, used for both imaging and nanomanipulation, which is the atomic force microscope (AFM) (Gerber and Lang, 2006). Therefore, first we will shortly review the applications of AFM in dentistry carried out by other groups in the past 20 years. Then, according to different topics of dentistry, we will review our own work in the area.

\section{AFM IN DENTISTRY}

Since its birth in the late 80 s, the AFM has progressively expanded its use from physical systems toward biological and biomedical applications (Kasas and Thomson, 1997). The plus of AFM is that samples require little preparation: no conductive coating is necessary, different from the scanning tunneling microscope and the scanning electron microscope (SEM), and no thin slices have to be cut such as in the transmission electron microscope (TEM). Instead, real 3D surfaces can be measured directly, even on bulky objects for stand-alone AFM heads. Furthermore, the environment can be ambient air, different from SEM/TEM, which usually work in high vacuum, or even liquid, which is the type of medium preferred by biological matter ${ }^{1}$.

For these reasons, and particularly for the non-destructive imaging, researchers have started to use AFM also in the field of dentistry since the mid-90s. The earliest works aimed to inspect the native dental tissue, especially dentin, after acid treatment (Marshall et al., 1993). In Buzalaf et al. (2014), the profile of mice teeth enamel crystals as responding to fluorosis was investigated, instead. An interesting comparison of AFM vs. SEM imaging of dentin was made in Kubinek et al. (2007), who pointed out the presence of artifacts due to dehydration in SEM. In particular, dehydration makes the collagen fibers in the tubules to collapse, which may be critical for the adhesion to dentin of the restorative composites. A difference in local mechanical properties surrounding the tubules has been observed by AFM in Kinney et al. (1996),

\footnotetext{
${ }^{1}$ In fact, this promise for easy operation often makes non-expert end-users think that practically every sample can be put under the AFM, whereas still basic requirements exist: (1) the sample must be a stable solid, i.e., not too gelly or sticky and properly bonded to the substrate; (2) the features must be sparse to be spatially resolved, and with the substrate appearing in between as the reference height, since AFM measures step-heights; (3) AFM is intrinsically slow and low in amount of contents, different from, e.g., SEM: indeed, even recent fast-scanning AFMs require acquisition times of minutes, and one AFM image allows for limited zoom-in since it typically contains $\sim 0.3$ vs. $\sim 1.2$ megapixels of SEM and $\geq 3$ of digital photography.
} 
who found fourfold hardness $(\sim 2.3 \mathrm{GPa})$ for peritubular vs. intertubular dentin. The mechanical properties of dental materials as measured by AFM have been the focus of a work by Pustan and Belcin (2009), who demonstrated also the measurement of frictions coefficient.

The effect of demineralization after exposure to different acids, for example, contained in beverages, has been often investigated, both in dentin (Eliades et al., 1999; Silikas et al., 1999) and in enamel (Pyne et al., 2009), in the latter work by real-time fast AFM. Conversely, the protective effect against enamel demineralization offered by a paste of casein phosphopeptides (Poggio et al., 2009) as well as different recent formulation toothpastes (Lombardini et al., 2014) has also been investigated.

Despite the limitation of AFM on samples formed by large and curved objects (see discussion in Section "Implant Surface Morphology"), it has been used successfully also on implants and orthodontic brackets wires (Silikas et al., 2001). Ceramics are still other dental materials on which AFM has been applied successfully, to characterize their surface modifications after laser (Folwaczny et al., 1998) or etching (Luo et al., 2001) or heat treatment (Gatin et al., 2013). Finally, AFM has proven useful also for fractographic analysis of failed surfaces in restorative composites (Jandt, 1998).

\section{DENTAL RESTORATIVE MATERIALS}

One major field of application of microscopy and nanotechnology to dentistry is in the characterization and fabrication of dental restorative composites, both in surface morphology and elastic properties. In recent years, several composites claimed to be "nano," such as Filtek Supreme (FS) by 3M-ESPE (3M-ESPE, 2014) and Venus diamond (VD) by Kulzer (2014). The latter in particular is an advanced formulation of hybrid composite, using filler particles with multiple size populations across both the microand the nano-scale (Ferracane, 2011), and was often chosen in our works as a reference material.

\section{SURFACE CHARACTERIZATION OF RESTORATIVE COMPOSITES}

The most advanced tool of surface characterization in nanotechnology is the AFM (Vahabi et al., 2013) along with the many other scanning probe microscopes derived from it (Cricenti et al., 2011). This instrument relies on physical contact of a very sharp tip with the specimen. As such, it can provide a direct measurement of 3D surfaces in the real space.

In Salerno et al. (2010a,b), we investigated the effect of airpolishing (AP) on VD restorative composite as a reference. The outcome of this analysis was the confirmation, as suggested by the counseling dentists that AP in itself does not only remove plaque but also damages the surface of composite-based dental restorations. This was assessed by measuring the change in the most common parameter of surface roughness, namely the root mean square (RMS) of heights, $S_{\mathrm{q}}$, after AP. The combinations of AP conditions among two abrading powders (bicarbonate and glycine), two jet distances ( 2 and $7 \mathrm{~mm})$, and three times $(5,10$, and $30 \mathrm{~s})$ were investigated. Even if the conditions for best AP treatment (i.e., least damage) were identified, still some level of damage is unavoidable (see Figures $\mathbf{2 A , B}$ ). This can have a consequence on long-term success of the restorations, due to recurring secondary caries, arising from the increased bacterial adhesion occurring on AP roughened surfaces.

In addition to measuring the dental surface damage and finding optimized AP conditions to limit it, in Salerno et al. (2010a,b), we also speculated on the possibility that an advanced AFM image analysis can correlate the surface damage to fractal dimensions, which could be used as a possible tool for screening of the treatment (see Figure 2C). In fact, surfaces both untreated and treated with maximum roughening conditions presented a fractal behavior, exhibiting self-affinity over the whole scale range accessible by the AFM, approximately from $300 \mathrm{~nm}$ to $100 \mu \mathrm{m}$ scan size. This is roughly three orders of magnitude, which is a rule-ofthumb minimum requirement for a surface to have fractal nature. When the fractal dimensions were calculated, the above surfaces (non-treated and badly treated) showed comparable values of 2.42.7. We concluded that the random non-optimized AP does not affect the fractal character of the surface. Differently, the surfaces treated with minimum roughening conditions lost their native fractal character, exhibiting no self-affinity.

In the mentioned study, both the experimental tool of AFM and the image analysis using fractal concepts came to the dental field from nanotechnology and advanced analytical practices of nanoengineering and nanoscience. Additional multifractal analysis carried out recently has confirmed the results of the basic fractal study (Tălu et al., 2015).

In another work (Salerno et al., 2012a,b), we studied the $S_{\mathrm{q}}$ roughness of dental restorative materials after another process similar to AP, which is polishing for dental restoration finishing. This polishing is applied to the dental surfaces not to remove the plaque and refresh their surfaces, but rather soon after their restoration, to decrease the surface roughness of the excess material used by the dentist to fill the dental cavity. As compared to AP, more massive and harder matter is milled during polishing, which requires solid abrading tools such as rotating grommets and burs. In this case, the starting surfaces are rougher with respect to the treated ones (see Figure 3C). The smoothest surface obtained is the best, same as for AP, for both medical (decrease of bacterial adhesion) and esthetical reasons (improve optical properties as reflectance and translucency).

In this study, not only the roughness was assessed but also the surface effects on the elastic properties of the materials were also investigated (Figures 3A,B. The null hypothesis was that the polishing would not cause degradation to the mechanical properties of the surface. To test this, we used a nanoindenter (Hay, 2009). As compared to old-fashioned microindenter, where only the final conditions of tip imprint size and maximum load reached are recorded, in nanoindentation [or instrumented indentation (Cripps and Anthony, 2004)] the datapoints at all intermediate instants of different load and penetration depth are measured during the experiment. This makes it possible to collect a whole load-indentation dataset, which is converted into a stress-strain engineering plot (Figure 3A). By fitting this plot to physical models, mainly Oliver-Pharr (Oliver and Pharr, 1992), both the surface hardness of the specimen and the elastic modulus of the material can be retrieved. In Salerno et al. (2012a,b), five load-indentation cycles were carried out on the specimens at increasing maximum (final) load between 40 and $200 \mathrm{mN}$ (Figure 3A). From each cycle, 

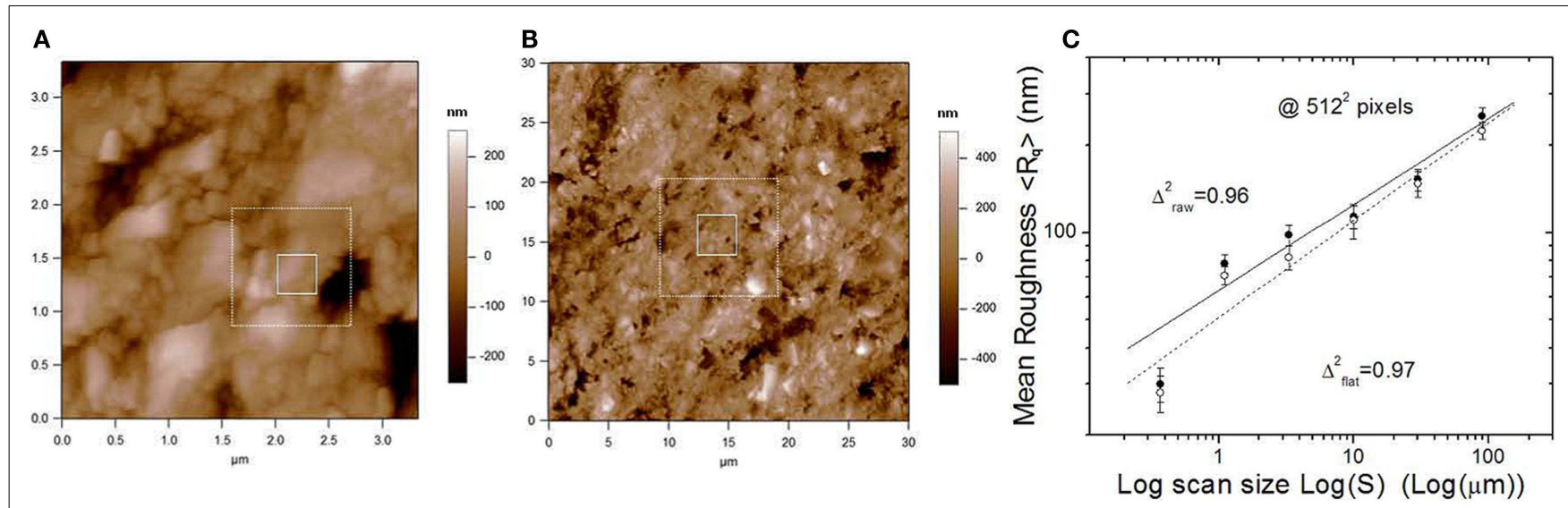

FIGURE 2 | (A,B) Representative AFM height images of VD restoration surface after $A P$, at (A) $3.33 \mu \mathrm{m}$ and (B) $30 \mu \mathrm{m}$ scan size, extracted form a sequence of $0.33-90 \mu \mathrm{m}$ scan size in steps of geometric ratio $\sim 3$ (see the white squares as a guide to progressive zooming regions). (C) Logarithmic plot of RMS roughness obtained from the images vs. the scan size: when a straight fits well enough the trend over the whole scan size range, the surface is fractal and the slope describes the fractal dimension. Reprinted from Salerno et al. (2010a).

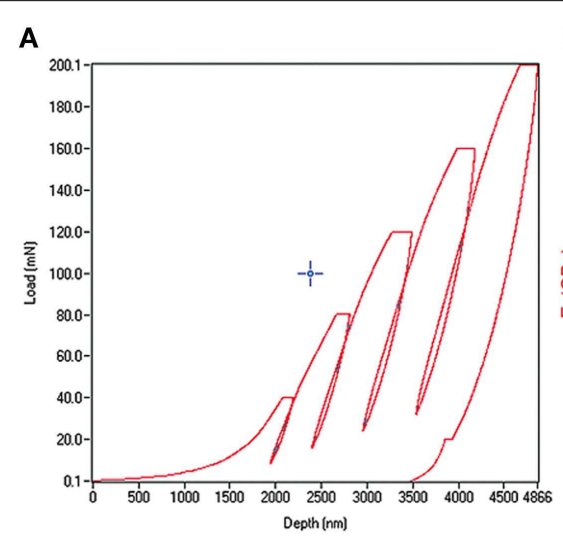

B

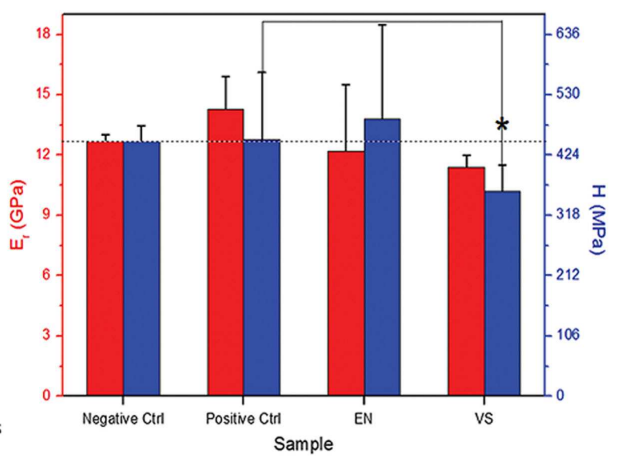

C

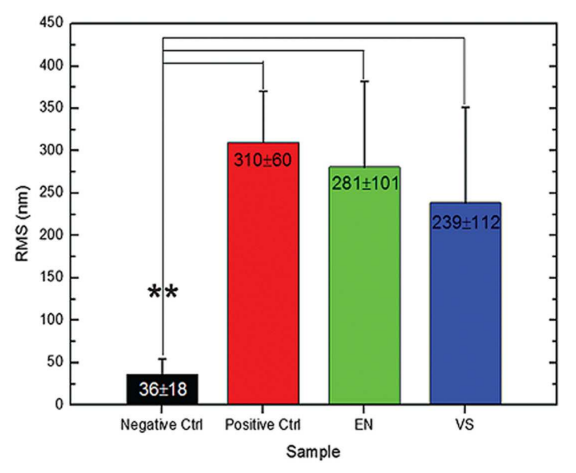

FIGURE 3 | (A) Typical nanoindentation curves of load vs. indentation, in this case, five cycles at increasing maximum (final) load have been carried out. (B) By fitting the curves in (A) to appropriate contact model (Oliver-Pharr here), values of hardness $\mathrm{H}$ and elastic modulus $\mathrm{E}$ can be obtained. (C) RMS roughness values resulting from AFM images of the polished restorative material. Reprinted from Salerno et al. $(2012 a, b)$. the apparent elastic modulus was calculated, resulting in a profile of elasticity at different depths in the polished material. At the less deep indentations of 1-2 $\mu \mathrm{m}$ (Figure 3B), a lower modulus was observed than that found at $5 \mu \mathrm{m}$ depth. In the latter case, depth was in excess of the surface roughness and the smear layer of polishing debris left on the surface, so the true elastic modulus was measured. Finally, the null hypothesis was confirmed that the elastic modulus is an intrinsic property of the material bulk.

In this work, not only the AFM again together with the nanoindenter have been taken from the "menu" of nanotechnological techniques. The analysis of surface composition was also addressed with advanced research tools of chemistry and physics. In fact, only the use of scanning electron microscopy (SEM) combined with chemical microanalysis by energy-dispersive spectroscopy (EDS) made it possible to identify the smear layer of polishing contamination products responsible for the apparent decrease in contact stiffness at the lowest indentation depths.
Another work across the morphological analysis of the surface and the mechanical elastic properties of the dental composites was carried out in Salerno et al. (2011). There, materials belonging to the class of flowable composites (Walter, 2013) were studied. The reference material for comparison was again VD, and the elastic properties were measured with the nanoindenter. The microscopic analysis by both AFM and SEM was the most important tool in this experiment, allowing to correlate the material properties to peculiar morphology of the matrix-filler distribution of the composites. In fact, we observed higher elastic modulus for the composites with higher filler particles loading, as expected. In particular, it was confirmed that AFM and SEM can be especially useful when used in combination. The access to the internal filler particles was obtained not by slicing the composites, which would probably damage the microtome diamond blade when crossing the matrixfiller modulus discontinuity of 2-70 GPa. Instead, some external material layers were removed by surface grinding. The main result 
was the observation that, as claimed for the latest generation flowable materials, at least in one case [Vertise Flow by Kerr (2014)] a flowable composite appeared enough stiff to be used also in bulk dental restorations.

The importance of SEM, in combination with AFM or as a faster alternative when no 3D surface topography is required, was confirmed in Salerno (2012b). This work focused on the standard restorative composite VD. With the support of EDS, we confirmed that the elasticity of the material was uniform inside the cured composite, same as qualitatively probed by AFM on a grinded section, due to material homogeneity. In fact, the elemental composition of the composite was uniform across the internal area exposed by grinding, which showed that the fillers, while well distinguished from the morphological point of view, were prepolymerized particles made of the same material surrounding them. Thus, the hybrid character of the composite (showing fillers of different size) was also confirmed in this study.

\section{ELASTIC PROPERTIES OF RESTORATIVE COMPOSITES}

Whereas the elastic properties of dental restorative composites have been traditionally investigated by standard tensile and compressive tests, in the past decade, application of nanoindentation has been increasingly spreading. This technique makes it possible to provide a more comprehensive view of the material, for example, the ratio of elastic-to-plastic behavior may be obtained, as well as the time-dependency of creep, when staying at a given maximum load and monitoring the changes in indentation. In this respect, in the last decade, AFM has become the nanotechnology standard for elastic measurements at high accuracy and spatial resolution, by means of force-spectroscopy of force-volume modes (Cappella and Dietler, 1999; Salerno et al., 2012a,b). On the other hand, for time-dependent viscous response and thanks to the chance of changing the working temperature (see Figures 4A,B) dynamic mechanical analysis [DMA (Menard, 1999)] has been increasingly used in the field of dental materials (Ryou et al., 2011).

Atomic force microscope and DMA have been used jointly in Thorat et al. (2012) (Figure 4C), to investigate the elastic modulus of experimental restorative composites based on fillers of titania, nanosilica, and milled glass. These composites were studied in a simplified model version, without the use of a coupling agent bonding the fillers and the matrix. AFM had already been used in Salerno (2012b) to measure the contact stiffness (see Figure 5), but in this work (Salerno, 2012a) the authors moved on to quantitative evaluation of the elastic modulus, which is known to be a critical step (Salerno, 2012b). Concurrently, DMA was used for cross-checking the AFM values of modulus, and a good agreement was observed between the two techniques (Figure 4C). As a consequence, we decided to always rely on the combined use of AFM and DMA for future characterizations of the elastic properties of dental materials.

On the way to the development of coupling-agent-free novel dental restorative materials, in Thorat et al. (2013a,b), we passed on from vitreous filler materials to hard metal oxides such as titania and alumina. For materials characterization, AFM and DMA were used as established previously. In Thorat et al. (2012), the nanosilica filler performed better than microscale ball-milled glass. Similarly, in this work, the nanoscale alumina was found to perform better than microscale alumina. This confirmed that the nano-size fillers provide some advantage with respect to microscale fillers of similar materials, probably due to the higher interfacial area to the matrix, when properly dispersed.

In a more recent work, the authors tried to exploit the nanoscale structuring of the filler particles for both mechanically reinforcing the material and for replacing the missing coupling-agent. To this goal, nanoporous microparticles of alumina have been engineered (Thorat et al., 2014). The controlled nanoporosity in alumina is obtained by anodization of an aluminum foil according to optimized conditions of voltage, electrolyte acid concentration, and bath temperature. As a result, a self-organized coating material called anodic porous alumina is obtained (Shingubara, 2003; Salerno et al., 2009). This material can be set free in the form of a self-standing membrane by etching away the remaining metal substrate. The alumina membranes were further processed by ball-milling, resulting into microscale particles with similar width as the thickness of the original membrane, in the range of $5-10 \mu \mathrm{m}$. The elastic modulus was measured by both AFM and DMA, before and after accelerated aging obtained with an equivalent thermal treatment of the specimens. It was observed that the filler micro-particles of ball-milled porous alumina provided better elastic modulus stability than two commercial restorative materials used for comparison. Obviously, a mechanical interlocking effect occurred thanks to the nanopores, which were infiltrated by the resin matrix during the mixing assisted by sonication. The found procedures were used to file a patent, before publication in the scientific literature (Salerno et al., 2014).

\section{DENTAL IMPLANTS}

Another important field of application of materials nanoscience and nanotechnology to dentistry is related to the characterization and development of dental implants. In our group, we have started to work with dental implants only few years ago, and so far we focused on the preliminary issue of the characterization of commercial implants. Given the well-assessed mechanical properties of the materials currently used, which are titanium (Ti) alloys and ceramics, presently the most required characterization work deals with advanced imaging of the implant surfaces and interfaces. The interfaces of interest in dental implants occur between the tooth ceramic crown on the top, the cement joining the crown to the metallic ( $\mathrm{Ti}$ alloy) parts of the abutment, and the implant itself (i.e., the screw hidden in the maxillar bone).

\section{IMPLANT SURFACE MORPHOLOGY}

In a recent work on dental implants (Cresti et al., 2013), we focused on $3 \mathrm{D}$ imaging of the interface between the abutment and the ceramic crown, namely the region of cement bonding. Because the recessed interface was generally not addressable by the comparatively short $(15 \mu \mathrm{m})$ AFM probe tip due to too large $Z$ range, a stylus profilometer was used. A small dedicated software was written in Igor (Wavemetrics, USA; Salerno, 2013), allowing to reassemble into $2 \mathrm{D}$ maps the $1 \mathrm{D}$ profile scans typical of the instrument. Different from AFM, the profilometer presented intrinsic imaging asymmetry, since the pixel size was of the order of $5 \mu \mathrm{m}$ between scan lines and $100 \mathrm{~nm}$ along the scan. Nevertheless, the rendering allowed one to identify reference marks made 
A

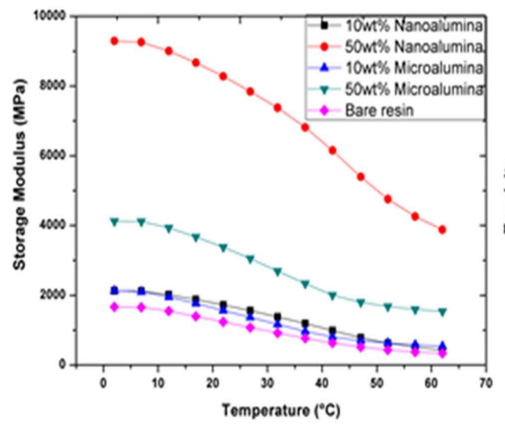

B
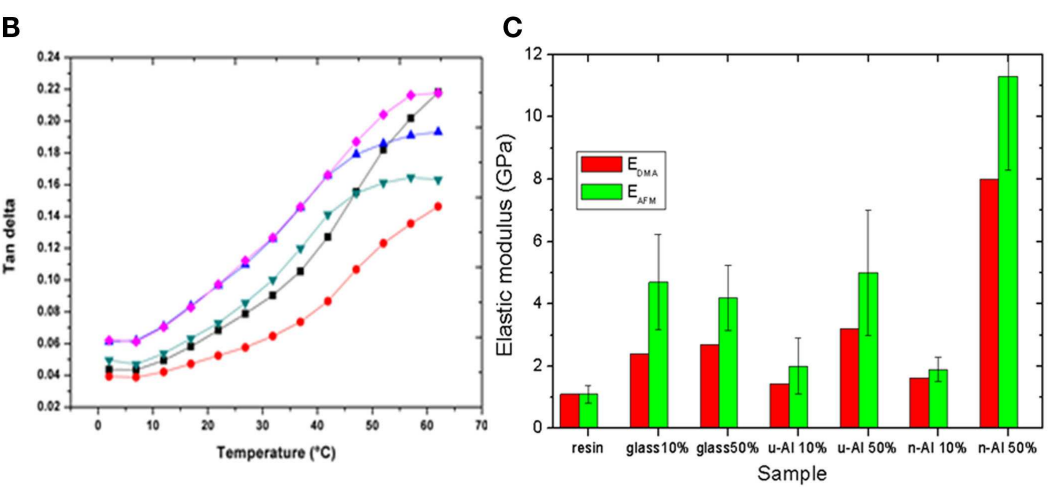

FIGURE 4 | (A,B) Typical response of DMA at $1 \mathrm{~Hz}$ (similar to mastication frequency), showing the values at the different temperatures scanned in the "oral" range of icy drinks to hot soups of both $(\mathbf{A})$ the elastic part of the complex modulus of the specimen material and (B) the viscous part (loss tangent, i.e., ratio of complex viscous modulus to real elastic modulus). In (C), the values of elastic modulus as obtained from DMA in (A) (RT value) are compared to the modulus obtained by AFM force-distance nanoindentation. Reprinted from Thorat et al. (2013a).

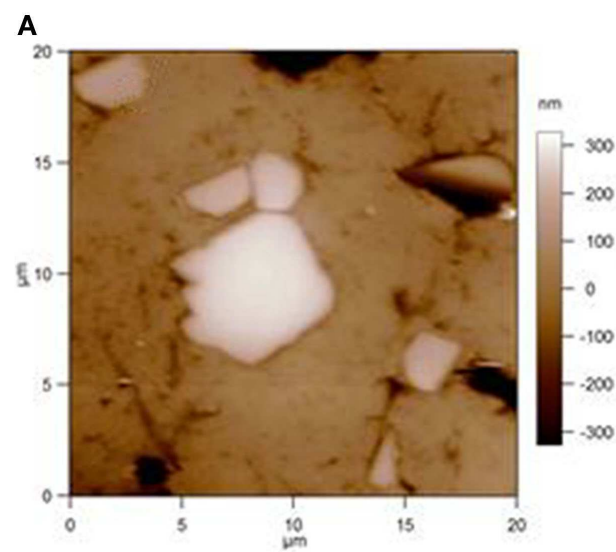

FIGURE 5 | (A) AFM height image and (B) AFM compliance image (after some thermal drift) of the surface of an experimental restorative composite including micro-alumina fillers. The bottom-left small bright spot in (B) is a void in the surface. In (C), the histogram
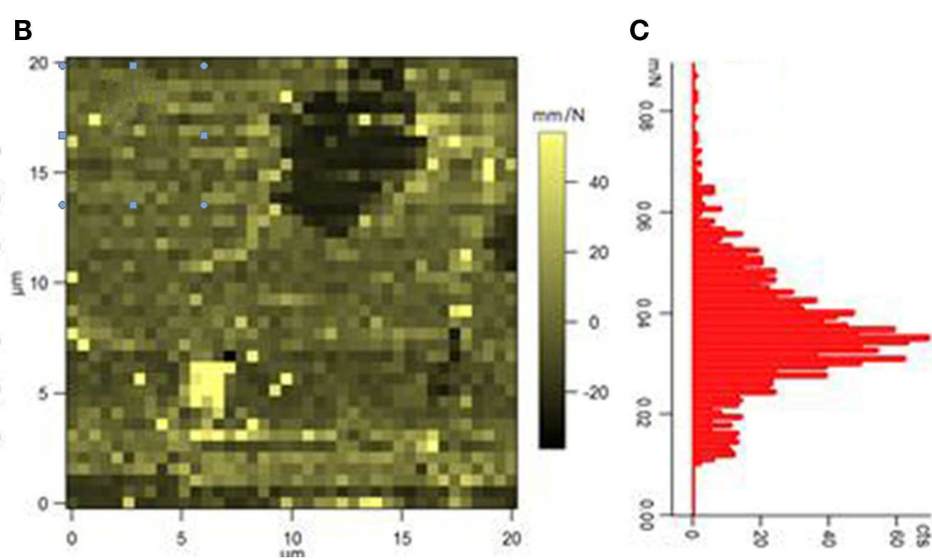

by indentation on the metal. Additionally, the 3D images obtained made it possible to use the standard techniques of AFM image processing to identify and isolate the cement region. To this goal, a $3 \times 3$ kernel filter based on a Sobel differential operator was used (Russ, 1998). Thus, we calculated the morphological parameters selected for description of the optimal interface, such as a step height at the materials edges, the width of cement (Cresti et al., 2013).

In a more recent work (Salerno et al., 2015), the possible morphological damage occurring on the surface of implant screws was evaluated, in terms of change of the surface after in vitro implantation tests in a model bone similar to the typical clinical bone [D1, Mish 90 classification (Misch, 1999)]. Within some limitations (only the top of the screw apex being accessible), AFM has been used in that case. The analysis of images pointed out relevant change in morphology and faced with the issue of its quantification. It should be stressed that having a change in the implant of compliance values as resulting from $(\mathbf{B})$ is plotted, showing a roughly bimodal behavior [the lowest peak at low values being associated to the stiff, filler, dark spot in top-right of (B)]. Reprinted from Thorat et al. (2013a). surface would not necessarily mean a decrease of performance, as in some instances the modified surface may even perform better than the original one. In any case, assessing the effect of insertion on the effective surface morphology and stability (i.e., possible delamination of coatings) is important not to misinterpret the success or failure of the implant.

Some researchers have recently experimented with implants based on metals other than $\mathrm{Ti}$, such as zirconium and tantalum. Notably, a controlled roughness, similar to that usually made on medical grade Ti by means of either acid etching or sand-blasting, can also be obtained with the anodization treatment used to make anodic porous alumina. In fact, Ti is another so-called valve metal same as $\mathrm{Al}$ (Salerno, 2014). In particular, the surface of $\mathrm{Ti}$, when anodized, tends to form adjacent nanotubes rather than nanopores joined by connected oxide walls (Uttiya et al., 2014). These porous titania nanotubes have been investigated so far mainly for applications in optics and photonics, thanks to the photocatalytic activity 
of titania (Mor et al., 2006). However, they could also be used as the surface modification of Ti implants in dentistry and orthopedics, similar to APA (Salerno, 2012b) and without the need for preliminary Al coating.

\section{IMPLANT SURFACE FUNCTIONALIZATION}

When the surface pattern of implants is endowed with nanopores, it obviously offers the chance of loading these pores with nanoparticles or biomolecules, for subsequent interaction with the surrounding living tissue. For APA, which has already been widely tested in the literature for biocompatibility with different cellular types (Poinern et al., 2011; Salerno et al., 2013; Toccafondi et al., 2014), loading of the pores has already been demonstrated with bioactive materials such as peptides (Swan et al., 2005), remineralization ions (Okawa et al., 2009), or antibacterial nanoparticles (Thorat et al., 2013a,b). In the latter work, the APA was loaded with nanoparticles of silver (see Figures 6A,B), which is a well-known biocide. Limited loading levels were reached (up to maximum $6 \%$ volume), which would still exhibit a significant pharmaceutical effect, with sustained release during a time longer than 2 days (Figure 6C). This experiment has been repeated in conditions of APA sealed under a coating of the same resin base as that used in our novel restorative composite of Thorat et al. (2014). Also, in this case, an effective release of silver was observed in a solution of phosphate buffer saline. In fact, while decreasing the levels of released silver, the sealing delays and thus extends the elution to a significantly longer time, of up to $\sim 5$ days. This type of functionalization for drug-delivery applications (Gultepe et al., 2010) could be the future goal also for anodization patterned porous $\mathrm{Ti}$ of implants.

Concerning drug delivery and bioactivity of nanotechnological materials, a number of products already exist in the market for general medicine applications. For example, Acticoat ${ }^{\mathrm{TM}}$ and Algisite $^{\mathrm{TM}}$ are patches for wound dressing based on antibacterial silver nanoparticles (Smith and Nephew, 2014). For products loaded instead with calcium phosphate or hydroxyapatite (HA) nanoparticles, Ostim ${ }^{\mathrm{TM}}$ [Osartis, Germany (Doessel and Schlegel, 2010)], Vitoss ${ }^{\mathrm{TM}}$ [Orthovita, USA (Kurien et al., 2013)], and NanOss ${ }^{\mathrm{TM}}$ [Angstrom Medica, USA (Roveri and Iafisco, 2010)] can be mentioned, which have been launched in recent years as synthetic bone graft substitutes. For specific dental applications, some HA coatings of dental implants have also been introduced [e.g., Spline Twist MP-1 ${ }^{\text {TM }}$ by Zimmer (2014), USA], but generally without much success probably due to delamination effects at the interface with the Ti alloy implant core.

\section{ADDITIONAL DENTAL APPLICATIONS OF NANOTECHNOLOGY: MOLECULAR SPECTROSCOPY}

So far, we have focused on the capability of nanotechnology to characterize the dental materials morphologically and mechanically - by ultra-resolution microscopy and advanced nanoprobe measurements - and to modify them - by nanostructuring and functionalization. In addition to this, for advanced characterization, a number of nanoscience techniques can also provide information on the composition and structure of materials, which fall under the general name of spectroscopy. A general advantage of spectroscopic techniques as compared to other testing methods is the non-invasiveness on the specimens. One example of spectroscopy has already been given with EDS. Additional common spectroscopic techniques are X-ray diffraction, used to identify the structure of crystalline inorganic nanoparticles (e.g., titania nanoparticles of HA) and also of biomolecules such as proteins and nucleic acids (Valdré et al., 1995); X-ray photoelectron spectroscopy, for chemical analysis of surface composition with part per thousand routine sensitivity (Kang et al., 2009); and vibrational molecular spectroscopy such as Raman scattering and Fouriertransformed infrared spectroscopy (FTIR) (Czichos et al., 2006). FTIR is already widely used in assessing the degree of conversion of the photopolymerized restorative resin composites, often in association with elastic measurements (Sideridou and Karabela, 2009; Thorat et al., 2012). Similarly, in a recent experiment, we used FTIR on dental impression materials to monitor their time response after mixing the base and catalyst. Thus, we could retrieve quantitative information on setting and working time of four impression materials, three belonging to the class of polyvinyl-siloxanes (VPS) and one being a conventional polyether (work under review). As compared to techniques such as shark fin test and Shore hardness durometer, FTIR can give a deeper insight into the chemistry

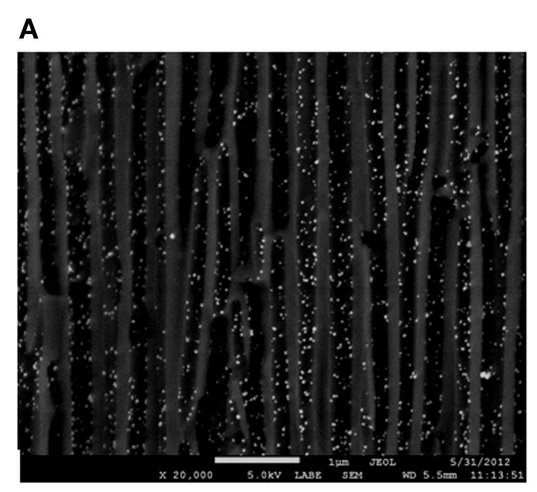

B



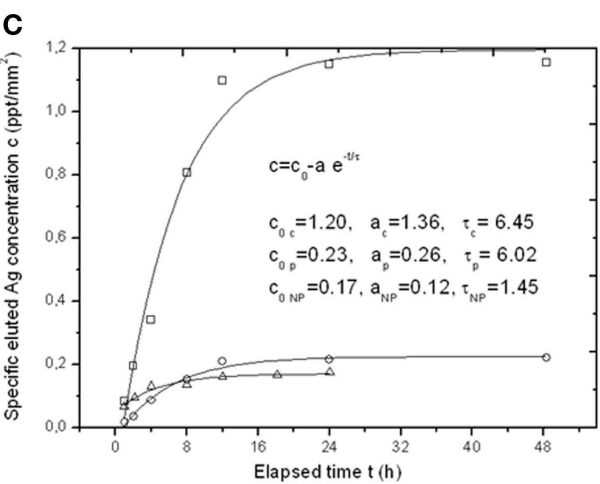

FIGURE 6 | (A) compositional SEM image (backscattered electrons) of the cross-section of an APA membrane as that used in the novel restorative composite of Thorat et al. (2014), showing high-atomic number nanoparticles dispersed in the pores. (B) SEM EDS spectra obtained from the same area as in (A), displaying the silver of the nanoparticles in (A). (C) Plots of elution of silver from loaded APA membranes as in (A,B). Reprinted from Thorat et al. (2013b). 
behind the decrease in viscosity and increase in stiffness of these elastomers during the setting. In fact, the spectral peaks describing the decrease in $\mathrm{Si}-\mathrm{H}$ bonds upon increase of the $\mathrm{Si}-\mathrm{O}$ ones during the setting have been identified in two materials, allowing to describe not only the setting kinetics but also their chemistry behind it. However, since the dental impression elastomers are complex materials that contain a number of components such as filler particles (to tune the rheology), color pigments, plasticizers, initiators, etc., the exact picture is difficult to obtain in the absence of detailed information available from the manufacturers.

Additional information may be obtained by complementary technique such as Raman scattering, since some molecular vibration modes are active in Raman and not (or not as sensitive) in FTIR, and vice-versa (Czichos et al., 2006). To be noted, both FTIR and Raman can today be coupled with optical microscope mapping, allowing spatial resolution on sample surfaces down to micrometer size spot (Mariani et al., 2010).

\section{CONCLUSION AND OUTLOOK}

In this work, some results obtained during the past few years in the field of dental material science, both in the literature and particularly in our institute, have been reviewed. The background of our group in microscopy and microfabrication techniques has been employed to obtain the presented results, and we are still struggling to pursue the application of nanoscience and nanotechnology practices further in the field of dental sciences. We are confident that this attitude of increasing use of modern inspection and manufacturing techniques also in dentistry will proceed at a faster pace in the next few years. Especially, the use of scanning probe microscopy and advanced light irradiation techniques, and possibly combinations thereof, can be foreseen as a field of future development. Laser treatment has already entered the dental practice, both as drill replacement and for speed-up activation of peroxide bleaching solution during teeth whitening. The use of laser, in connection with photocatalytic materials such as titania, has also been proposed for disinfection (Riley et al., 2005). More recently, fluorescence probing has been introduced for detection of oral cancer (Shin et al., 2010). Similarly for AFM, endoscopic instruments imaging dental surfaces in situ in totally non-invasive manner have also been postulated (Stolz et al., 2007). In this application, the AFM could also be used as a nanoscalpel, or empowered with light irradiation to make real-time in vivo biosensors based on tip-enhanced Raman spectroscopy.

We find that there are no major technological barriers against this cross-contamination of research and technology between different areas of physics, engineering, and dentistry, but for the psychological barriers from different education of dental doctors. The point of view of dentists and major dental companies will have to change, and the latter in particular should open wider to this scenario and invest significantly in the field. A relatively small portion of the budget currently devoted to advertisement and promotion of dental products could actually foster the development of new practices and products of possibly great benefit to the clinical results. However, a shift is required in the mind of both dental practitioners and dental companies to take full advantage of nanoscience and nanotechnology, which for the dental area has so far remained mainly in the academia.

\section{REFERENCES}

3M-ESPE. (2014). Filtek Supreme. Available at: http://www.3m.com/3M/en_US/ Dental/Products/Catalog/ /Filtek-Supreme-Ultra-Universal-Restorative?N= $5145652+3294736391 \& \mathrm{rt}=$ rud

American Dental Association. (2014). Glossary of Dental Clinical and Administrative Terms. Available at: http://www.ada.org/glossaryforprofessionals.aspx

Zimmer. (2014). Anon Zimmer Dental Inc. 1900 Aston Avenue Carlsbad, CA 92008, USA (760) 929-471 4300. Available at: http://www.zimmerdental.com/Home/ zimmerDental.aspx

Buzalaf, M. A., Barbosa, C. S., Leite Ade, L., Chang, S. R., Liu, J., Czajka-Jakubowska, A., et al. (2014). Enamel crystals of mice susceptible or resistant to dental fluorosis: an AFM study. J. Appl. Oral Sci. 22, 159-164. doi:10.1590/1678775720130515

Cappella, B., and Dietler, G. (1999). Force-distance curves by atomic force microscopy. Surf. Sci. Rep. 34, 1-104. doi:10.1016/S0167-5729(99)00003-5

Cresti, S., Itri, A., Rebaudi, A., Diaspro, A., and Salerno, M. (2013). Microstructure of titanium-cement-lithium disilicate interface in CAD-CAM dental implant crowns: a three-dimensional profilometric analysis. Clin. Implant Dent. Relat. Res., 4-6. Available at: http://www.ncbi.nlm.nih.gov/pubmed/23968260

Cricenti, A., Colonna, S., Girasole, M., Gori, P., Ronci, F., Longo, G., et al. (2011) Scanning probe microscopy in material science and biology. J. Phys. D Appl. Phys. 44, 464008. doi:10.1088/0022-3727/44/46/464008

Cripps, F., and Anthony, C. (2004). Nanoindentation. New York, NY: Springer.

Czichos, H., Saito, T., and Smith, L. (eds) (2006). Springer Handbook of Materials Measurement Methods. Springer.

Doessel, O., and Schlegel, W. C. (eds) (2010). "World congress on medical physics and biomedical engineering September 7-12, 2009 Munich, Germany," in Biomaterials, Cellular and Tissue Engineering, Artificial Organs, Vol. 25/X (Springer Science and Business Media).

Eliades, G., Vougiouklakis, G., and Palaghias, G. (1999). Effect of dentin primers on the morphology, molecular composition and collagen conformation of aciddemineralized dentin in situ. Dent. Mater. 15, 310-317. doi:10.1016/S01095641(99)00050-0

Ferracane, J. L. (2011). Resin composite - state of the art. Dent Mater. 27, 29-38. doi:10.1016/j.dental.2010.10.020

Folwaczny, M., Mehl, A., Haffner, C., and Hickel, R. (1998). Polishing and coating of dental ceramic materials with $308 \mathrm{~nm} \mathrm{XeCl}$ excimer laser radiation. Dent. Mater. 14, 186-193. doi:10.1016/S0109-5641(98)00029-3

Freitas, R. (2000). Nanodentistry. J. Am. Dent. Assoc. 131, 1559-1565. doi:10.14219/ jada.archive. 2000.0084

Gatin, E., Luculescu, C., Iordache, S., and Patrascu, I. (2013). Morphological investigation by AFM of dental ceramics under thermal processing. J. Optoelectron. Adv. Mater. 15, 1136-1141.

Gerber, C., and Lang, H. P. (2006). How the doors to the nanoworld were opened. Nat. Nanotechnol. 1, 3-5. doi:10.1038/nnano.2006.70

Gultepe, E., Nagesha, D., Sridhar, S., and Amiji, M. (2010). Nanoporous inorganic membranes or coatings for sustained drug delivery in implantable devices. $A d v$. Drug Deliv. Rev. 62, 305-315. doi:10.1016/j.addr.2009.11.003

Hay, J. (2009). Introduction to instrumented indentation testing. Exp. Tech. 33, 66-72. doi:10.1063/1.2830028

Jandt, K. D. (1998). Structure of microfilled dental composite fractured surfaces. Probe Microsc. 1, 323-331.

Kang, B. S., Sul, Y. T., Oh, S. J., Lee, H. J., and Albrektsson, T. (2009). XPS, AES and SEM analysis of recent dental implants. Acta Biomater. 5, 2222-2229. doi:10.1016/j.actbio.2009.01.049

Kasas, S., and Thomson, N. (1997). Biological applications of the AFM: from single molecules to organs. Int. J. Imaging Syst. Technol. 8, 151-161. doi:10.1002/(SICI) 1098-1098(1997)8:2<151::AID-IMA2>3.0.CO;2-9

Kerr. (2014). Vertise Flow. Available at: http://www.kerrdental.com/ kerrdental-composites-vertiseflow-2

Kinney, J. H., Balooch, M., Marshall, S. J., Marshall, G. W. Jr., and Weihs, T. P. (1996). Atomic force microscope measurements of the hardness and elasticity of peritubular and intertubular human dentin. J. Biomed. Eng. 118, 133-135.

Kubinek, R., Zapletalova, Z., Vujtek, M., Novotný, R., Kolarova, H., and Chmelickova, H. (2007). Examination of dentin surface using AFM and SEM. Dent. Mater. 593598. Available at: http://www.formatex.org/microscopy3/pdf/pp593-598.pdf

Kulzer, H. (2014). Venus Diamond. Available at: http://www.heraeus-venus.com/it/ it/products_7/venusdiamond_4/venusdiamond_1.html 
Kumar, S. R., and Vijayalakshmi, R. (2006). Nanotechnology in dentistry. Indian J. Dent. Res. 17, 62-65. doi:10.4103/0970-9290.29890

Kurien, T., Pearson, R. G., and Scammell, B. E. (2013). Bone graft substitutes currently available in orthopaedic practice: the evidence for their use. Bone Joint $J$. 95-B, 583-597. doi:10.1302/0301-620X.95B5.30286

Lombardini, M., Ceci, M., Colombo, M., Bianchi, S., and Poggio, C. (2014). Preventive effect of different toothpastes on enamel erosion: AFM and SEM studies. Scanning 36, 401-410. doi:10.1002/sca.21132

Luo, X. P., Silikas, N., Allaf, M., Wilson, N. H. F., and Watts, D. C. (2001). AFM and SEM study of the effects of etching on IPS-Empress 2 dental ceramic. Surf. Sci. 491, 388-394. doi:10.1016/S0039-6028(01)01301-2

Mariani, M. M., Day, P. J. R., and Deckert, V. (2010). Applications of modern micro-Raman spectroscopy for cell analyses. Integr. Biol. (Camb) 2, 94-101. doi:10.1039/b920572a

Marshall, G. W. Jr., Balooch, M., Tench, R. J., Kinney, J. H., and Marshall, S. J. (1993). Atomic force microscopy of acid effects on dentine. Dent. Mater. 9, 265-268. doi:10.1016/0109-5641(93)90072-X

Menard, K. P. (1999). Dynamic Mechanical Analysis, A Practical Introduction. Boca Raton, NY: CRC Press.

Misch, C. E. (1999). Contemporary Implant Dentistry. St. Louis, MO: Mosby.

Mor, G. K., Varghese, O. K., Paulose, M., Shankar, K., and Grimes, C. A. (2006). A review on highly ordered, vertically oriented $\mathrm{TiO} 2$ nanotube arrays: fabrication, material properties, and solar energy applications. Sol. Energ. Mater. Sol. Cell. 90, 2011-2075. doi:10.1016/j.solmat.2006.04.007

Okawa, S., Homma, K., Kanatani, M., and Watanabe, K. (2009). Characterization of calcium phosphate deposited on valve metal by anodic oxidation with polarity inversion. Dent. Mater. J. 28, 513-518. doi:10.4012/dmj.28.513

Oliver, W. C., and Pharr, G. M. (1992). An improved technique for determining hardness and elastic modulus using load and displacement sensing indentation experiments. J. Mater. Res. 7, 1564. doi:10.1557/JMR.1992.1564

Poggio, C., Lombardini, M., Dagna, A., Chiesa, M., and Bianchi, S. (2009). Protective effect on enamel demineralization of a CPP-ACP paste: an AFM in vitro study. J. Dent. 37, 949-954. doi:10.1016/j.jdent.2009.07.011

Poinern, G. E., Shackleton, R., Mamun, S. I., and Fawcett, D. (2011). Significance of novel bioinorganic anodic aluminum oxide nanoscaffolds for promoting cellular response. Nanotechnol. Sci. Appl. 4, 11-24. doi:10.2147/NSA.S13913

Pustan, M., and Belcin, O. (2009). Application of atomic force microscope for mechanical and tribological characterization of teeth and biomaterials. Tribology in industry. 31, 43-46.

Pyne, A., Marks, W., M Picco, L., G Dunton, P., Ulcinas, A., E Barbour, M., et al. (2009). High-speed atomic force microscopy of dental enamel dissolution in citric acid. Arch. Histol. Cytol. 72, 209-215. doi:10.1679/aohc.72.209

Riley, D. J., Bavastrello, V., Covani, U., Barone, A., and Nicolini, C. (2005). An in-vitro study of the sterilization of titanium dental implants using low intensity UV-radiation. Dent. Mater. 21, 756-760. doi:10.1016/j.dental.2005.01. 010

Roveri, N., and Iafisco, M. (2010). Evolving application of biomimetic nanostructured hydroxyapatite. Nanotechnol. Sci. Appl.3, 107-125. doi:10.2147/NSA.S9038

Russ, J. C. (1998). The Image Processing Handbook, 3rd Edn. CRC Press, Springer, IEEE Press.

Ryou, H., Niu, L. N., Dai, L., Pucci, C. R., Arola, D. D., Pashley, D. H., et al. (2011). Effect of biomimetic remineralization on the dynamic nanomechanical properties of dentin hybrid layers. J. Dent. Res. 90, 1122-1128. doi:10.1177/ 0022034511414059

Salerno, M. (2012a). Improved estimation of contact compliance via atomic force microscopy using a calibrated cantilever as a reference sample. Measurement 45 , 2103-2113. doi:10.1016/j.measurement.2012.05.011

Salerno, M. (2012b). Improved estimation of contact compliance via atomic force microscopy using a calibrated cantilever as a reference sample. Measurement 45 , 2103-2113. doi:10.1016/j.measurement.2012.05.011

Salerno, M. (2013). Procedure for Assembling DAT ASCII Files of 1 D Profiles from XP2 (Ambios-USA) into 2D Maps of Height. Available at: http://www.igorexchange. com/node $/ 2699$

Salerno, M. (2014). Introduction to the special issue on "nanostructures by valve metal anodization". J. Mater. Sci. Nanotechnol. 1(1):Se101. doi:10.15744/23489812.1.Se101

Salerno, M., Caneva-Soumetz, F., Pastorino, L., Patra, N., Diaspro, A., and Ruggiero, C. (2013). Adhesion and proliferation of osteoblast-like cells on anodic porous alumina substrates with different morphology. IEEE Trans. Nanobioscience 12, 106-111. doi:10.1109/TNB.2013.2257835

Salerno, M., Derchi, G., Thorat, S., Ceseracciu, L., Ruffilli, R., and Barone, A. C. (2011). Surface morphology and mechanical properties of new-generation flowable resin composites for dental restoration. Dent. Mater. 27, 1221-1228. doi:10.1016/j.dental.2011.08.596

Salerno, M., Diaspro, A., and Thorat, S. (2014). Combined Material Including Anodic Porous Alumina and a Polymer Matrix, and Its Use for the Dental Recondition. Available at: http://worldwide.espacenet.com/publicationDetails/ originalDocument? $\mathrm{CC}=\mathrm{WO} \& \mathrm{NR}=2014053946 \mathrm{~A} 1 \& \mathrm{KC}=\mathrm{A} 1 \& \mathrm{FT}=\mathrm{D} \& \mathrm{ND}=$ $3 \&$ date $=20140410 \& D B=E P O D O C \& l o c a l e=e n \_E P$

Salerno, M., et al. (2015). Surface microstructure of dental implants pre- and postinsertion: an in vitro study by means of scanning probe microscopy. Implant Dentistry. (in press).

Salerno, M., Giacomelli, L., Derchi, G., Patra, N., and Diaspro, A. (2010a). Atomic force microscopy in vitro study of surface roughness and fractal character of a dental restoration composite after air-polishing. Biomed. Eng. Online 9, 59. doi:10.1186/1475-925X-9-59

Salerno, M., Giacomelli, L., and Larosa, C. (2010b). Biomaterials for the programming of cell growth in oral tissues: the possible role of APA bioinformation. Bioinformation 5, 291-293. doi:10.6026/97320630005291

Salerno, M., Patra, N., and Cingolani, R. (2009). Use of ionic liquid in fabrication, characterization, and processing of anodic porous alumina. Nanoscale Res. Lett. 4, 865-872. doi:10.1007/s11671-009-9337-3

Salerno, M., Patra, N., and Diaspro, A. (2012a). Atomic force microscopy nanoindentation of a dental restorative midifill composite. Dent. Mater. 28, 197-203. doi:10.1016/j.dental.2011.10.007

Salerno, M., Patra, N., Thorat, S., Derchi, G., and Diaspro, A. (2012b). Combined effect of polishing on surface morphology and elastic properties of a commercial dental restorative resin composite. Sci. Adv. Mater. 4, 126-134. doi:10.1166/sam.2012.1261

Shin, D., Vigneswaran, N., Gillenwater, A., and Richards-Kortum, R. (2010). Advances in fluorescence imaging techniques to detect oral cancer and its precursors. Future Oncol. 6, 1143-1154. doi:10.2217/fon.10.79

Shingubara, S. (2003). Fabrication of nanomaterials using porous alumina templates. J. Nanopart. Res. 5, 17-30. doi:10.1023/A:1024479827507

Sideridou, I. D., and Karabela, M. M. (2009). Effect of the amount of 3methacyloxypropyltrimethoxysilane coupling agent on physical properties of dental resin nanocomposites. Dent. Mater. 25, 1315-1324. doi:10.1016/j.dental. 2009.03.016

Silikas, N., Lennie, A. R., England, K., and Watts, D. C. (2001). AFM as a tool in dental research. Microsc. Anal. 19-21.

Silikas, N., Watts, D. C., England, K. E., and Jandt, K. D. (1999). Surface fine structure of treated dentine investigated with tapping mode atomic force microscopy (TMAFM). J. Dent. 27, 137-144. doi:10.1016/S0300-5712(98) 00032-3

Smith and Nephew. (2014). Acticoat Product Line. Available at: http: //www.smith-nephew.com/uk/products/wound_management/product-search/ acticoat/

Stolz, M., Aebi, U., and Stoffler, D. (2007). Developing scanning probe-based nanodevices-stepping out of the laboratory into the clinic. Nanomedicine 3, 53-62. doi:10.1016/j.nano.2007.01.001

Swan, E. E. L., Popat, K. C., and Desai, T. A. (2005). Peptide-immobilized nanoporous alumina membranes for enhanced osteoblast adhesion. Biomate rials 26, 1969-1976. doi:10.1016/j.biomaterials.2004.07.001

Tălu, S, Stachb, S., Albc, S. F., and Salerno, M. (2015). Multifractal characterization of a dental restorative composite after air-polishing. Chaos Solitons Fractals 71, 7-13. doi:10.1016/j.chaos.2014.11.009

Thorat, S., Diaspro, A., and Salerno, M. (2013a). Effect of alumina reinforcing fillers in BisGMA-based resin composites for dental applications. Adv. Mater. Lett. 4, 15-21. doi:10.5185/amlett.2013.icnano. 283

Thorat, S., Diaspro, A., Scarpellini, A., Povia, M., and Salerno, M. (2013b). Comparative study of loading of anodic porous alumina with silver nanoparticles using different methods. Materials 6, 206-216. doi:10.3390/ma6010206

Thorat, S., Patra, N., Ruffilli, R., Diaspro, A., and Salerno, M. (2012). Preparation and characterization of a BisGMA-resin dental restorative composites with glass, silica and titania fillers. Dent. Mater. J. 31, 635-644. doi:10.4012/dmj. 2011-251 
Thorat, S. B., Diaspro, A., and Salerno, M. (2014). In vitro investigation of couplingagent-free dental restorative composite based on nano-porous alumina fillers. J. Dent. 42, 279-286. doi:10.1016/j.jdent.2013.12.001

Toccafondi, C., Thorat, S., La Rocca, R., Scarpellini, A., Salerno, M., Dante, S., et al. (2014). Multifunctional substrates of thin porous alumina for cell biosensors. J. Mater. Sci. Mater. Med. 25, 2411-2420. doi:10.1007/s10856-014-5178-4

Uttiya, S., Contarino, D., Prandi, S., Carnasciali, M. M., Gemme, G., Mattera, L., et al. (2014). Anodic oxidation of titanium in sulphuric acid and phosphoric acid electrolytes. J. Mater. Sci. Nanotechnol. 1, S106.

Vahabi, S., Nazemi Salman, B., and Javanmard, A. (2013). Atomic force microscopy application in biological research: a review study. Iran. J. Med. Sci. 38, 76-83.

Valdré, G., Mongiorgi, R., Monti, S., Corvo, G., Itro, A., Paroli, R., et al. (1995). [Xray powder diffraction (XRD) in the study of biomaterials used in dentistry. 3]. Minerva Stomatol. 44, 21-32.

Walter, R. (2013). Bulk-fill flowable composite resins. J. Esthet. Restor. Dent. 25 , 72-76. doi:10.1111/jerd.12011
Conflict of Interest Statement: The authors declare that the research was conducted in the absence of any commercial or financial relationships that could be construed as a potential conflict of interest.

Received: 10 January 2015; accepted: 26 February 2015; published online: 18 March 2015.

Citation: Salerno M and Diaspro A (2015) Dentistry on the bridge to nanoscience and nanotechnology. Front. Mater. 2:19. doi: 10.3389/fmats.2015.00019

This article was submitted to Nanobiotechnology, a section of the journal Frontiers in Materials.

Copyright (c) 2015 Salerno and Diaspro. This is an open-access article distributed under the terms of the Creative Commons Attribution License (CC BY). The use, distribution or reproduction in other forums is permitted, provided the original author(s) or licensor are credited and that the original publication in this journal is cited, in accordance with accepted academic practice. No use, distribution or reproduction is permitted which does not comply with these terms. 